Annals of Warsaw University of Life Sciences - SGGW

Land Reclamation No 38, 2007: 25-32

(Ann. Warsaw Univ. of Life Sci. - SGGW, Land Reclam. 38, 2007)

\title{
Load of heavy metals in drainage waters in the Middle Sudety Mountains
}

\author{
KRZYSZTOF PULIKOWSKI, STANISŁAW KOSTRZEWA, JULIAN PALUCH, \\ SZYMON SZEWRAŃSKI \\ Institute of Environmental Development and Protection, \\ Wrocław University of Environmental and Life Sciences
}

\begin{abstract}
Load of heavy metals in drainage waters in the Middle Sudety Mountains. Research were carried out in study site located in Stare Bogaczowice near Wałbrzych. The daily drainage runoff measurements and periodical chemical analyses of water samples from selected drainage plots had been made. Concentrations of heavy metals: $\mathrm{Cu}, \mathrm{Zn}, \mathrm{Zn}, \mathrm{Pb}$, $\mathrm{Cr}$ i Cd had been measured. Drainage waters do not include significant amounts of heavy metals. Concentration of zinc, which is very common in nature, ranged from 0.018 to $0.675 \mathrm{mg} \mathrm{Zn} \cdot \mathrm{dm}^{-3}$, cadmium concentrations was somewhat larger then $0.001 \mathrm{mg} \mathrm{Cd} \cdot \mathrm{dm}^{-3}$. In spite of precipitations higher then in the other sites and because of low concentrations, the heavy metal loads had been very small. Regarding lead and nickel load did not exceed $70 \mathrm{~g} \cdot \mathrm{ha}^{-1} \cdot \mathrm{yr}^{-1}$, and concerning copper, chromium and cadmium it was below $5.0 \mathrm{~g} \cdot \mathrm{ha}^{-1}$. $\cdot \mathrm{yr}^{-1}$. Heavy metals in drainage waters do not make serious threat on surface water body receivers.
\end{abstract}

Key words: heavy metals, load, draining, water.

\section{INTRODUCTION}

The water movement through a soil profile is strongly connected with process of mineral and organic substances leaching. In the most cases leaching seems to be unfavourable. Continual leaching tends to impoverish the soil profile as well as to ground and surface water pollution. Exceptionally, the leaching could be useful for removing anthropogenic and natural pollutants from soil or ground profile [Kelleners, Chaudhry, 1998; Kelleners et al., 2000].

The heavy metals are also being leached and the intensity of this process depends on many factors such: metal form, soil type, alkalinity, infiltration rate and others.

The aim of research was to specify the concentrations of selected heavy metals in drainage waters and determine the leaching yield of metals transported with waters to reservoir.

\section{MATERIALS AND RESEARCH METHODS}

The study site is located in borderland of the Bolków-Watbrzych Foothills and Wałbrzych Mountains in the region of the Middle Sudety. Research was carried out in the hydrological years 1996/1997, $1997 / 1998$ and $1998 / 1999$ on 19.04 ha of drained arable lands. Concentration of heavy metals was determined in waters flowing from 4 drainage systems with area range from 1.03 to 1.87 ha. Part of drainage systems is made with pipe spacing of 11 meters (drainage plots 1 and 11), in plots 3 and 4 doubled spacing was applied. Drainage pipes are laid at 
depth of 0.95 meter in every drainage plot. The pseudo-podzols predominate in study site, their grain size composition is characteristic for silty and clay loam with large amounts of solid particles with diameter bigger then $1 \mathrm{~mm}$. Soil profiles are rather shallow $(1.2 \mathrm{~m})$ and located above the parent rock rubble.

The mean annual precipitation in study site according to IMGW Szczawno Zdrój meteorological point is $655 \mathrm{~mm}$, the mean air temperature is $7.3^{\circ} \mathrm{C}$. Higher precipitations appeared in two years during investigations and in the year 1998/1999, total precipitation was lower - $595 \mathrm{~mm}$ (Tab. 1). In July 1997 the neighbouring areas had been flooded but the study site, as located higher, wasn't under floodwaters. Drainage water discharge was measured by filling the container of known volume - from $0.5 \mathrm{dm}^{3}$ to $10 \mathrm{dm}^{3}$, depending on water runoff. Water samples had been collected into plastic bottles, directly from drainage outlets. Sampling was performed once a month, when system was draining the waters. Zinc, coper, nickel, lead, chromium and cadmium were been determined with ASA method. The daily metal loads had been calculated from the daily runoff volume and components concentrations in water. Regarding that chemical analyses had been carried out once a month, linear interpolation for

TABLE 1. Specification of days with drainage runoff during a year $(\mathrm{Tg})$, the maximum daily runoff yield (qmax) runoff depth $(\mathrm{H})$, total precipitations $(\mathrm{P})$ and runoff coefficients $(\alpha)$

\begin{tabular}{|c|c|c|c|c|c|c|}
\hline 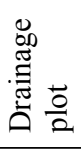 & Years & $\underset{\left[\mathrm{dm}^{3} \cdot \mathrm{s}^{-1} \cdot \mathrm{ha}^{-1}\right]}{\max }$ & $\begin{array}{c}\mathrm{H} \\
{[\mathrm{mm}]}\end{array}$ & $\begin{array}{c}\mathrm{P} \\
{[\mathrm{mm}]}\end{array}$ & $\begin{array}{c}\alpha=\frac{H}{P} \\
{[\%]}\end{array}$ & $\mathrm{P}-\mathrm{H}$ \\
\hline \multirow{4}{*}{1} & $1996 / 1997$ & 2.32 & 284 & 889 & 31.9 & 605 \\
\hline & $1997 / 1998$ & 1.54 & 274 & 737 & 37.2 & 463 \\
\hline & $1998 / 1999$ & 0.93 & 87 & 595 & 14.7 & 508 \\
\hline & mean & - & 215 & 740 & 29.1 & 525 \\
\hline \multirow{4}{*}{3} & $1996 / 1997$ & 1.34 & 176 & 889 & 19.8 & 713 \\
\hline & $1997 / 1998$ & 1.34 & 212 & 737 & 28.8 & 525 \\
\hline & 1998/1999 & 1.78 & 193 & 595 & 32.4 & 402 \\
\hline & mean & - & 194 & 740 & 26.2 & 546 \\
\hline \multirow{4}{*}{4} & $1996 / 1997$ & 1.77 & 118 & 889 & 13.3 & 771 \\
\hline & $1997 / 1998$ & 0.20 & 79 & 737 & 10.7 & 658 \\
\hline & 1998/1999 & 0.71 & 68 & 595 & 11.4 & 527 \\
\hline & mean & - & 88 & 740 & 11.9 & 652 \\
\hline \multirow{4}{*}{11} & $1996 / 1997$ & 2.43 & 290 & 889 & 32.3 & 599 \\
\hline & $1997 / 1998$ & 1.21 & 306 & 737 & 41.5 & 431 \\
\hline & $1998 / 1999$ & 2.42 & 197 & 595 & 33.1 & 398 \\
\hline & mean & - & 264 & 740 & 35.7 & 476 \\
\hline
\end{tabular}


intervals was applied. The annual total load ( $\mathrm{E}$ ) running off from 1 ha unit area of drainage plot, was calculated as a sum of the daily loads from 1st November to 31 st October in the nextyear [Pulikowski, 2004]:

$\mathrm{L}=\sum_{\mathrm{t}=1}^{\mathrm{t}=365}\left(\overline{\mathrm{Q}}_{\mathrm{t}} \cdot \mathrm{C}_{\mathrm{t}}^{\mathrm{I}}\right)$

where:

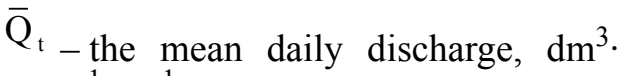
$\cdot$ day $^{-1} \cdot \mathrm{ha}^{-1}$,

$\mathrm{C}_{\mathrm{t}}^{\mathrm{I}}$ - the mean daily concentration, for days with no data, concentration is determined from linear interpolation, $\mathrm{mg} \cdot \mathrm{dm}^{-3}$,

$\mathrm{t}-$ following day.

\section{RESEARCH RESULTS AND DISCUSSION}

Pipe draining is commonly used for improving air-water conditions on agricultural lands, specially on arable grounds. The outflow volume of drainage water, mainly in early spring, depends on soil type, height and precipitation distribution, air temperature, slope and terrain shape, drain spacing, land use (arable, meadow), crop type [Kostrzewa, 1977;Pływaczyk, 1991].RegardingPolish conditions, the total rainfalls recorded in study site seem to be relatively high. These circumstances cause, that drainage system removes water much longer. The mean runoff depth, in specific drainage plots, is diverse and ranges from $68 \mathrm{~mm}$ (plot 4, year 1998/1999) to $306 \mathrm{~mm}$ (plot 11, year 1997/1998). These results could be explained by doubled drain spacing applied in plot 4 and surface topography.
Runoff coefficient in specific drainage plots ranges from 11.9 to $35.7 \%$, the mean for 4 plot is $25.7 \%$. Regarding presented values and assumption of $655 \mathrm{~mm}$ of the total rainfall, the mean annual runoff yield should reach 1683 $\mathrm{m}^{3} \cdot \mathrm{ha}^{-1}$. Runoff depth and coefficient are strongly connected with precipitation depth. Runoff depth is related directly to increasing precipitation, when runoff coefficient shows completely reverse indirect relationship. In effect, water storage range in the site is much smaller then annual precipitations range (Tab.1). Drainage plot 3 seems to be exceptional, where retention exceeds the total rainfalls. Obtained runoff coefficients are in the ranges cited in literature [Kostrzewa, 1977; Pływaczyk, 2002]. The maximum runoff yields exceed $2.4 \mathrm{dm}^{3} \cdot \mathrm{ha}^{-1} \cdot \mathrm{yr}^{-1}$.

Regarding 6 investigated metals, the highest concentration in waters was recorded for zinc. The mean concentration ranged from 0.018 to 0.675 $\mathrm{mg} \mathrm{Zn} \cdot \mathrm{dm}^{-3}$. The mean calculated for all 4 drainage plots was $0.212 \mathrm{mg} \mathrm{Zn} \cdot \mathrm{dm}^{-3}$ (Tabs 2, 3). Obtained results are higher then concentrations measured in drainage waters from sewage irrigated grounds [Kostrzewa et al., 1999]. The high zinc concentration is caused by its common occurrence in earth's crust as well as by its ability to form soluble compounds, which can be easily leached from soil profile by infiltrating waters [KabataPendias, Pendias, 1993]. The next trace elements $(\mathrm{Cu}, \mathrm{Ni}$ and $\mathrm{Cr})$ occurred in much lower amounts (Tabs 2,3). Quite often, especially regarding chromium, concentrations had been lower then the lowest measurement resolution of 0,001 $\mathrm{mg} \cdot \mathrm{dm}^{-3}$. Regarding lead and cadmium, currently recognized as completely 
TABLE 2. Heavy metal concentrations in drainage waters from the specific sections $\mathrm{mg} \cdot \mathrm{dm}^{-3}$

\begin{tabular}{|c|c|c|c|c|c|c|}
\hline & $\mathrm{Zn}$ & $\mathrm{Cu}$ & $\mathrm{Pb}$ & $\mathrm{Ni}$ & $\mathrm{Cd}$ & $\mathrm{Cr}$ \\
\hline 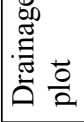 & \multicolumn{6}{|c|}{$\frac{\text { minimum }- \text { maximum }}{\text { mean }}$} \\
\hline 1 & $\frac{0.018-0.525}{0.187}$ & $\frac{<0.001-0.008}{0.002}$ & $\frac{<0.001-0.026}{0.010}$ & $\frac{0.001-0.051}{0.011}$ & $\frac{<0.001-0.001}{<0.001}$ & $\frac{<0.001-0.003}{0.001}$ \\
\hline 2 & $\frac{0.025-0.535}{0.282}$ & $\frac{<0.001-0.008}{0.002}$ & $\frac{<0.001-0.014}{0.005}$ & $\frac{<0.001-0.023}{0.006}$ & $<0.001$ & $\frac{<0.001-0.001}{0.001}$ \\
\hline 3 & $\frac{0.024-0.675}{0.184}$ & $\frac{<0.001-0.008}{0.002}$ & $\frac{<0.001-0.014}{0.005}$ & $\frac{<0.001-0.052}{0.010}$ & $<0.001$ & $\frac{<0.001-0.001}{0.001}$ \\
\hline 11 & $\frac{0.023-0.505}{0.214}$ & $\frac{<0.001-0.008}{0.002}$ & $\frac{<0.001-0.119}{0.024}$ & $\frac{<0.001-0.038}{0.13}$ & $<0.001$ & $\frac{<0.001-0.002}{0.001}$ \\
\hline
\end{tabular}

TABLE 3. Characteristic heavy metal concentrations in drainage waters $\mathrm{mg} \cdot \mathrm{dm}^{-3}$

\begin{tabular}{|l|c|c|c|c|c|c|}
\hline Specification & $\mathrm{Zn}$ & $\mathrm{Cu}$ & $\mathrm{Pb}$ & $\mathrm{Ni}$ & $\mathrm{Cd}$ & $\mathrm{Cr}$ \\
\hline Mean concentration & 0.212 & 0.002 & 0.012 & 0.010 & $<0.001$ & 0.001 \\
\hline Maximum concentration & 0.675 & 0.008 & 0.119 & 0.052 & 0.001 & 0.003 \\
\hline
\end{tabular}

useless in biochemical processes, concentrations had also been not high. Cadmium concentration exceeded significance level only in drainage plot 1 , a little bit higher records, up to 0.119 $\mathrm{mg} \mathrm{Pb} \cdot \mathrm{dm}^{-3}$, were been noticed for lead examination. Actually, this value was abnormal and could be considered as accidental regarding the average concentration range from $<0.001$ to $0.026 \mathrm{mg} \mathrm{Pb} \cdot \mathrm{dm}^{-3}$. Metal concentrations in drainage waters are similar to ones in leakages from lysimeters filled up with different soils [Ruszkowska et al., 1996].

Heavy metal concentrations in drainage waters, except zinc, are lower then amounts recorded in rainfall waters [Wardecka, 2004]. Lysimeter experiments on metal leaching also proved higher concentrations in rainwater then in leakages [Ruszkowska et al., 1996].
Chemical compounds load is mainly related to the total runoff volume. Study site is characterized by quite high runoff coefficient, which causes heavy metal loads higher then in the other regions.

Just only in the case of zinc, in years $1996 / 1997$ in plot 11, the annual total load exceeded $1 \mathrm{~kg} \mathrm{Zn} \cdot \mathrm{ha}^{-1} \cdot \mathrm{yr}^{-1}$ (Fig. 1). In individual years, meanly from 86 to $820 \mathrm{~g} \mathrm{Zn} \cdot \mathrm{ha}^{-1} \cdot \mathrm{yr}^{-1}$ flowed out from drainage plot. Value of copper runoff yield did not exceed $2.5 \mathrm{~g} \mathrm{Cu} \cdot \mathrm{ha}^{-1} \cdot \mathrm{yr}^{-1}$, and chromium $5.0 \mathrm{~g} \mathrm{Cr} \cdot \mathrm{ha}^{-1} \cdot \mathrm{yr}^{-1}$ (Figs 2 i 3). Compared to lisymeter experiments obtained results on copper are definitely lower. In experiment, metal amounts leached during one year, in relation to 1 ha, ranged from 13 to $22 \mathrm{~g} \mathrm{Cu} \cdot \mathrm{ha}^{-1} \cdot \mathrm{yr}^{-1}$ [Ruszkowska et al., 1996].

Because of similar concentrations of nickel and lead in examined waters, also their total loads seemed to be 


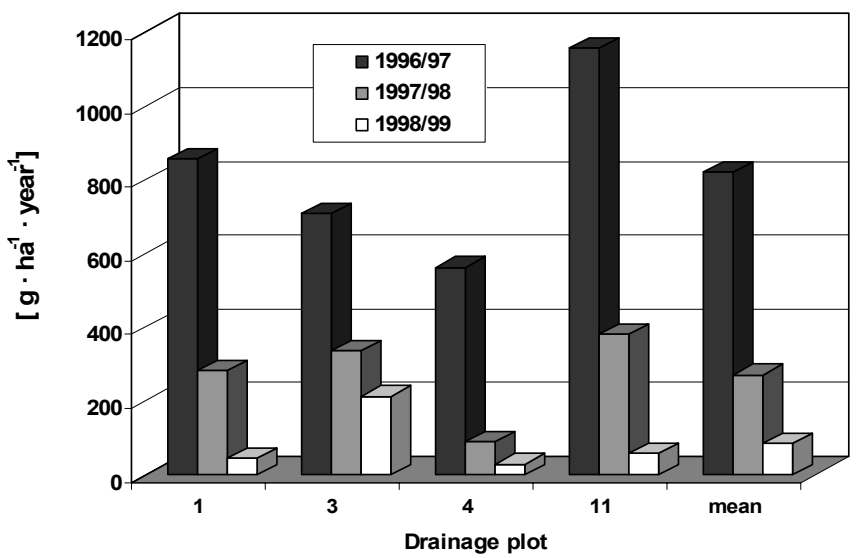

FIGURE 1. Zinc load in drainage waters

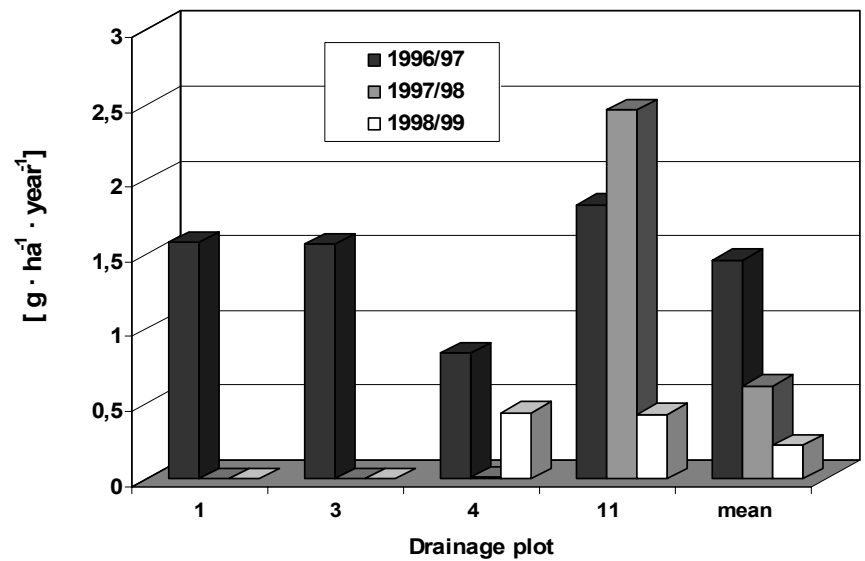

FIGURE 2. Copper load in drainage waters

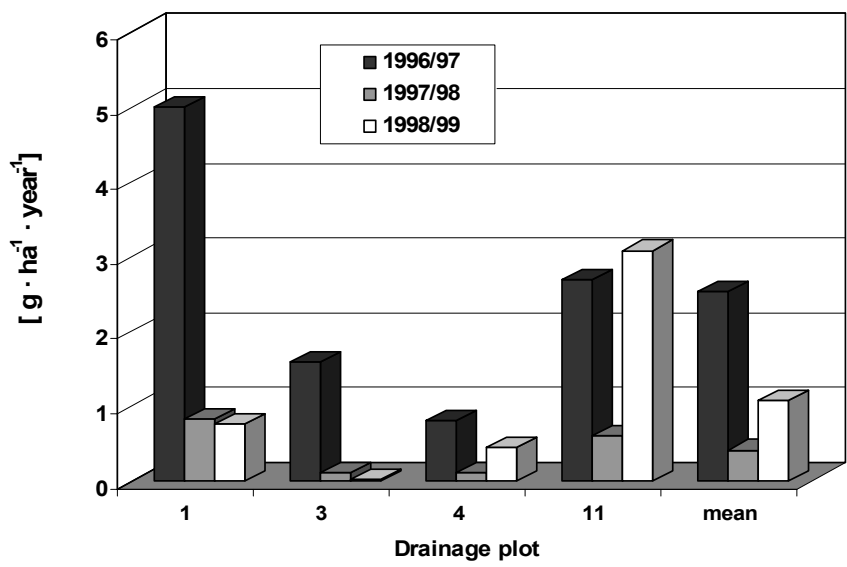

FIGURE 3. Chromium load in drainage waters 
comparable. The biggest lead load -64.2 $\mathrm{g} \mathrm{Pb} \cdot \mathrm{ha}^{-1} \cdot \mathrm{yr}^{-1}$ run out in year 1996/1997 from plot 11 (Fig. 4). The highest nickel load was noticed in plot 1 and reached $57.8 \mathrm{~g} \mathrm{Ni}^{\circ} \mathrm{ha}^{-1} \cdot \mathrm{yr}^{-1}$ (Fig. 5). The mean metal runoff yield during research period amounted only $12 \mathrm{~Pb} \cdot \mathrm{ha}^{-1} \cdot \mathrm{yr}^{-1}$ and 18.5 $\mathrm{g} \mathrm{Ni} \cdot \mathrm{ha}^{-1} \cdot \mathrm{yr}^{-1}$. The cadmium loads were
It could suggest, that soils had been able to accumulate cadmium that time. Considering portions delivered by rainfall waters, total metals input equals to the total loads transported in waters and carried with crops.

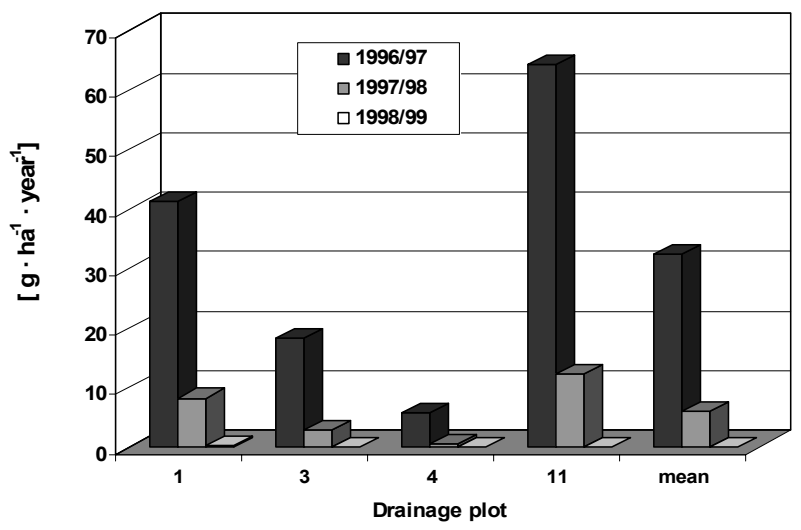

FIGURE 4. Lead load in drainage waters

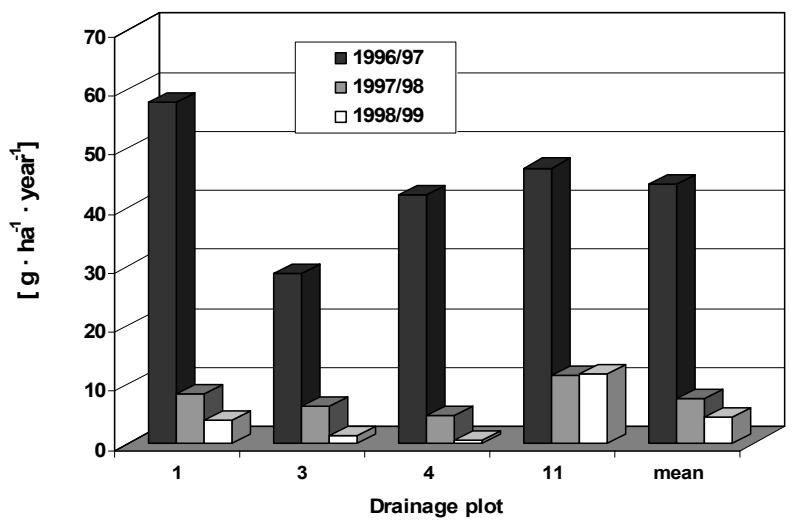

FIGURE 5. Nickel load in drainage waters

very small and exceeded $1.0 \mathrm{~g} \mathrm{Cd} \cdot \mathrm{ha}^{-1}$ per year in no cases. Presented values do not make serious threat to surface water quality. By Smal and Kot [2005] in years 1955-1995 about $1.9 \mathrm{~g} \mathrm{Cd} \cdot \mathrm{ha}^{-1} \cdot \mathrm{yr}^{-1}$ and $14.2 \mathrm{~g} \mathrm{~Pb} \cdot \mathrm{ha}^{-1} \cdot \mathrm{yr}^{-1}$ were been brought into soils by fertilizers and manures.

\section{CONCLUSIONS}

1. Drainage waters do not include significant amounts of heavy metals. Concentration of zinc, which is very common in nature, ranged from 0.018 to $0.675 \mathrm{mg} \mathrm{Zn} \cdot \mathrm{dm}^{-3}$, cadmium 
concentrations was somewhat larger then $0.001 \mathrm{mg} \mathrm{Cd} \cdot \mathrm{dm}^{-3}$;

2. In spite of higher precipitations and because of low concentrations, the heavy metal loads had been very small. Regarding lead and nickel load did not exceed $70 \mathrm{~g} \cdot \mathrm{ha}^{-1} \cdot \mathrm{yr}^{-1}$, and concerning copper, chromium and cadmium it was below $5.0 \mathrm{~g} \cdot \mathrm{ha}^{-1} \cdot \mathrm{yr}^{-1}$.

3. Heavy metals in drainage waters do not make serious threat on surface water body receivers.

\section{REFERENCES}

KELLENERS T.J. CHAUDHRY M.R. 1998: Drainage water salinity of tubewells and pipe drains: A case study from Pakistan. Agricultural Water Management 37: 41-53.

KELLENERS T.J., KAMRA S.K., JHORAR R.K. 2000: Predication of long term drainage water salinity of pipe drains. Journal of Hydrology 234: 249-236.

KOSTRZEWA S. 1977: Badania nad ustaleniem norm odpływu drenarskiego w terenach nizinnych południowo-zachodniej Polski. Zesz. Nauk. AR Wroc., Rozprawy 3: 61.

KOSTRZEWA S., PULIKOWSKI K., PALUCH J. 1999: Odpływ metali ciężkich z sieci drenarskiej do otwartych koryt cieków wodnych. Ochrona Środowiska i Zasobów Naturalnych nr 18: 271-279.

KABATA-PENDIAS A., PENDIAS H. 1993: Biogeochemia pierwiastków śladowych. PWN, Warszawa: 364.

PŁYWACZYK A. 1991: Skuteczność drenowania gruntów ornych terenów nizinnych i górskich na Dolnym Śląsku. Zesz. Nauk. AR Wroc., Rozprawy 91: 59.

PŁYWACZYK A. 2002: Wpływ urządzeń drenarskich na stosunki wodne gruntów ornych w terenach nizinnych Dolnego Śląska. Wyd. AR we Wrocławiu, 42.
PULIKOWSKI K. 2004: Zanieczyszczenia obszarowe w małych zlewniach rolniczych. Zesz. Nauk. AR we Wrocławiu, ser. Rozprawy CCXI, 479: 137.

RUSZKOWSKA M., KUSIO M., SYKUT S. 1996: Wymywanie pierwiastków śladowych z gleby w zależności od rodzaju i nawożenia (badania lizymetryczne). Roczn. Glebozn. T. XLVII nr 1/2: 11-22. SMAL H., KOT I. 2005: Szacunek ładunków $\mathrm{Cd}$ i $\mathrm{Pb}$ dostających się do gleb Polski w latach 1955-1995. Zesz. Probl. PNR Warszawa 505: 293-303.

WARDECKA L. 2004: Dynamika roztworu glebowego w warunkach naturalnych. Zesz. Nauk. AR we Wrocławiu, ser. Rolnictwo LXXXV, 487:183-209.

Streszczenie: Ladunek metali ciężkich odptywajacy $z$ wodami drenarskimi $w$ Sudetach Srodkowych. W pracy przedstawiono wyniki badań dotyczących zawartości wybranych metali ciężkich w wodach drenarskich i jednostkowego ładunku poszczególnych metali wynoszonych do odbiornika wraz z wodą. Badania prowadzono na obiekcie położonym na pograniczu Pogórza BolkowskoWałbrzyskiego i Gór Wałbrzyskich. Obejmowały one natężenie odpływu wód drenarskich i oznaczenie zawartości: cynku, miedzi, niklu, ołowiu, chromu i kadmu.

Średni roczny wskaźnik odpływu dla poszczególnych działów jest zróżnicowany i wynosi od $68 \mathrm{~mm}$ do $306 \mathrm{~mm}$. Z sześciu metali objętych badaniami w najwyższych stężeniach występował cynk. Jego średnie stężenie wynosiło od 0,018 do $0,675 \mathrm{mg} \mathrm{Zn} \cdot \mathrm{dm}^{-3}$, natomiast średnie obliczone dla 4 działów wyniosło $0,212 \mathrm{mg} \mathrm{Zn} \cdot \mathrm{dm}^{-3}$. Tak duże stężenie cynku wynika z jego powszechnego występowania w skorupie ziemskiej, jak również tworzenia przez ten metal związków łatwo rozpuszczalnych. Pozostałe mikroelementy $(\mathrm{Cu}$, $\mathrm{Ni}, \mathrm{i} \mathrm{Cr}$ ) występowały w znacznie mniejszych ilościach.

Wielkość ładunku metali ciężkich, jak i innych składników wynoszonych wraz $\mathrm{z}$ wodami drenarskimi jest uzależniona głównie od ilości odpływającej wody. Obiekt, na którym prowadzono badania, charakteryzował się wysokim wskaźnikiem odpływu. Jedynie w przypadku cynku w dziale 11 zanotowano roczny ładunek przekra- 
czający $1 \mathrm{~kg} \mathrm{Zn} \cdot \mathrm{ha}^{-1} \cdot \mathrm{rok}^{-1}$. W poszczególnych latach odpływało średnio od 86 do $820 \mathrm{~g} \mathrm{Zn} \cdot \mathrm{ha}^{-1}$. $\cdot$ rok $^{-1}$. Ilość miedzi odpływająca $\mathrm{z}$ jednego hektara w ciągu roku nie przekroczyła $2,5 \mathrm{~g} \mathrm{Cu} \cdot \mathrm{ha}^{-1}$. $\cdot$ rok $^{-1}$, a chromu 5,0 $\mathrm{g} \mathrm{Cr} \cdot \mathrm{ha}^{-1} \cdot \mathrm{rok}^{-1}$. Średnio w okresie badawczym odpływało $\mathrm{z} 1$ ha zaledwie około $12 \mathrm{~g} \mathrm{~Pb} \cdot \mathrm{ha}^{-1} \cdot \mathrm{rok}^{-1}$ i $18,5 \mathrm{~g} \mathrm{Ni} \cdot \mathrm{ha}^{-} 1 \cdot \mathrm{rok}^{-1}$. Ładunki kadmu były bardzo małe i w żadnym $\mathrm{z}$ analizowanych przypadków nie przekroczyły $1,0 \mathrm{~g} \mathrm{Cd} \cdot \mathrm{ha}^{-1} \cdot \mathrm{rok}^{-1}$. Ładunki metali ciężkich zawarte $\mathrm{w}$ wodach drenarskich nie stanowią zagrożenia dla wód powierzchniowych stanowiących ich odbiornik.
MS. received December 2007
Authors' address:
Krzysztof Pulikowski
50-363 Wrocław, pl. Grunwaldzki 24
Institute of Environmental Development and Protection
Wrocław University of Environmental and Life Sciences
krzysztof.pulikowski@up.wroc.pl 